\title{
latrogenic Spinal Epidural Hematoma in the Acute Postoperative Period
}

\author{
Roxanna Rasekhi, DO, ${ }^{1}$ Omar Viswanath, MD, ${ }^{2}$ Eugene Fu, MD, ${ }^{1}$ Carla Cordova, MD ${ }^{1}$ \\ ${ }^{1}$ University of Miami, School of Medicine, Department of Anesthesiology, Perioperative Medicine and Pain Management, University \\ of Miami Health System, Jackson Memorial Hospital, Miami, FL ${ }^{2}$ Department of Anesthesia, Critical Care, and Pain Medicine, \\ Beth Israel Deaconess Medical Center, Boston, MA
}

Background: Spinal epidural hematomas (SEHs) are a rare but serious complication associated with spinal surgery, with an incidence estimated to be as high as $3 \%$. Patients present with back pain and neurologic symptoms, with a typical onset time of several hours to days following surgery.

Case Report: A 60-year-old female who underwent cervical spine surgery had negative intraoperative neuromonitoring and no apparent neurologic deficits of the upper extremities immediately postsurgery. On arrival in the postanesthesia care unit, the patient reported a loss of sensation in her lower extremities, prompting the anesthesiology resident to perform a focused neurologic examination. The patient demonstrated 0/5 motor strength in the lower extremities and total loss of sensation to touch and temperature up to the level of T10 bilaterally. She was returned to the operating room, and surgical exploration revealed a cervical SEH. Decompression yielded abrupt return of motor evoked potentials and improvement in somatosensory evoked potentials.

Conclusion: As the primary clinician in the perioperative period, the anesthesiologist is ideally positioned to be the first to detect any deterioration in a patient's condition. Anesthesiologists should be cognizant of the potential for acute presentation of SEH so that it can be considered in the differential diagnosis.

Keywords: Anesthesia recovery period, hematoma-epidural-spinal, neurosurgery, perioperative care, postoperative care

Address correspondence to Carla Cordova, MD, Department of Anesthesiology, Perioperative Medicine and Pain Management, UHealth Tower, 1400 NW 12th Ave., 3rd Floor, Suite 3075, Miami, FL 33136. Tel: (305) 689-1106. Email: ccordova@med.miami.edu

\section{INTRODUCTION}

Postoperative spinal epidural hematoma (SEH) is a rare but serious complication of spinal surgery, and rapid detection is vital to the prevention of significant morbidity and mortality. The incidence of symptomatic postoperative SEH requiring surgical evacuation has been cited to be as high as $0.1 \%-3.0 \%$, with most sources citing a more conservative incidence near $0.1 \%-1.0 \% .^{1-3}$ Because this complication is not commonly seen, it can easily be missed and often is not included in the clinician's differential diagnosis. Risk factors include age $>60$ years, alcohol consumption of more than 10 drinks per week, preoperative use of nonsteroidal antiinflammatory drugs, a history of spinal surgery, and spinal surgeries at multiple levels. ${ }^{1,4}$

Patients with SEH may initially present with symptoms of back pain and neurologic deficits. Typically, the onset of neurologic deficits secondary to SEH development occurs in hours or days, with a maximum onset of 8 days. ${ }^{4} \mathrm{~A}$ retrospective study examining 4,568 spinal operations at the $\mathrm{Na}$ tional Hospital for Neurology and Neurosurgery and The Wellington Hospital in London, England, found that the median onset of initial symptoms for all SEHs was 2.7 hours. ${ }^{4}$
Given the long onset time of symptoms, coupled with the low incidence of $0.1 \%-3.0 \%$, caregivers may overlook this serious complication in the acute postoperative period.

\section{CASE REPORT}

A 60-year-old female with a medical history of hypertension and gastroesophageal reflux presented with severe cervical radiculopathy following a work-related injury. She was scheduled for $\mathrm{C} 4-\mathrm{C} 7$ corpectomies with discectomies and anterior fusion. After standard induction and endotracheal intubation, neuromonitoring leads were placed for the intermittent monitoring of electromyography (EMG), motor evoked potentials (MEPs), and somatosensory evoked potentials (SSEPs). Maintenance of anesthesia was done with total intravenous anesthetic consisting of propofol and remifentanil. The fusion was successfully completed, and the patient maintained reassuring tracings on EMG, MEPs, and SSEPs throughout the surgery. The patient was extubated in the operating room (OR), and initial postoperative examination revealed no apparent neurologic deficits of the upper extremities bilaterally. Because the patient had undergone a cervical surgery, the postoperative examiner only evaluated her upper extremities. 
During mobilization for transport to the postanesthesia care unit (PACU), the patient reported sharp subscapular back pain with radiation to both shoulders. Her back pain was initially attributed to prolonged surgical positioning with a shoulder roll. The patient was transported to the PACU, where she continued to report upper back discomfort and a loss of sensation in her legs. The anesthesiology resident performed a focused neurologic physical examination that revealed 0/5 motor strength in the lower extremities and a loss of sensation to temperature, touch, and pinprick up to the level of T10 bilaterally. The patient had no deficits in her upper extremities. The patient was extubated at 11:11 am and transported from the OR at 11:23 am. She arrived in the PACU at 11:35am. Her neurologic sequelae occurred within 24 minutes after extubation.

The neurosurgery team performed a neurologic examination in the PACU. Following confirmation of neurologic deficits, the patient was taken back to the OR, and standard monitors were placed prior to induction. Following rapid sequence induction, the patient was repositioned prone, and neuromonitoring leads were placed. Baseline MEPs of the abductor hallucis showed a total deficit of the lower extremities bilaterally. Surgical exploration revealed $200 \mathrm{~mL}$ of bloody fluid behind the level of C6. Decompression of the hematoma elicited abrupt return of MEPs and improvement in SSEPs (Figure). The patient had complete recovery following surgical evacuation and reported no issues at her 1- and 6-month follow-ups.

\section{DISCUSSION}

After thorough review, we were unable to find any reports of a cervical SEH presenting only with lower extremity symptoms, which is likely why the initial postoperative examiner did not perform a full-body neurologic examination. ${ }^{3-6}$ Despite normal EMG, MEPs, and SSEPs throughout the initial surgery, this patient had a serious surgical complication with symptoms that began before she left the OR. The patient's cervical $\mathrm{SEH}$ developed at a rate much quicker than has been de-

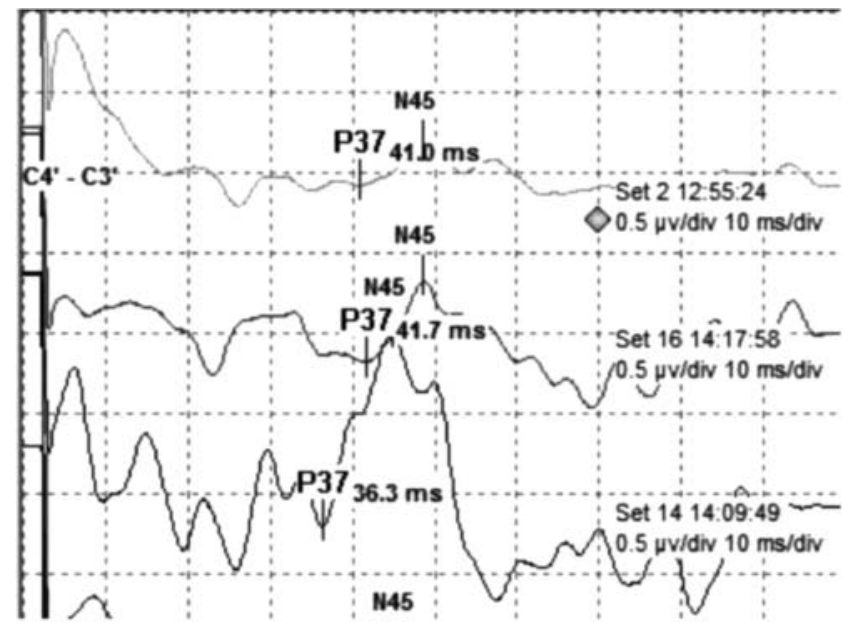

Figure. The baseline somatosensory evoked potential (SSEP) tracing from the beginning of the reoperation (top) showed minimal responsiveness, but a dramatic return of SSEPs occurred immediately following surgical decompression (bottom). scribed in the literature, with preservation of upper extremity function. ${ }^{4}$ Rapid recognition led to a quick decision to return to the OR, thus preventing any permanent neurologic deficits.

The patient's first postoperative neurologic examination was incomplete, and her complaints of midthoracic pain were initially dismissed. Normal intraoperative neuromonitoring may have given all parties a false sense of security regarding the patient's postoperative presentation. Upon the discovery of motor and sensory deficits, the first consideration was possible conversion disorder, a psychiatric condition brought on by emotional stress, in which patients demonstrate a loss of neurologic function that is not explained by any identifiable medical condition. ${ }^{7}$

Patients must be closely monitored in the postoperative period to ensure early recognition of any rapidly developing neurologic deficits. The prognosis of patients who develop postoperative SEH primarily depends on the timely evacuation of the hematoma. Because the prognosis of $\mathrm{SEH}$ is time dependent, being aware of the speed at which these hematomas can develop and how quickly they can progress is important. The greatest neurologic recovery is seen in patients who undergo evacuation within 6 hours of diagnosis, with better outcomes for those who undergo evacuation sooner.,

\section{CONCLUSION}

As the primary clinician in the perioperative period, the anesthesiologist is ideally positioned to be the first to detect any deterioration in a patient's condition. The preponderance of postoperative SEHs present several hours to days following surgery, but the anesthesiologist should be cognizant of the potential for acute presentation and perform a thorough neurologic evaluation as soon as possible. This case highlights that anesthesiologists must practice with a questioning attitude and perform due diligence throughout all phases of perioperative care.

\section{ACKNOWLEDGMENTS}

The authors have no financial or proprietary interest in the subject matter of this article.

\section{REFERENCES}

1. Awad JN, Kebaish KM, Donigan J, Cohen DB, Kostuik JP. Analysis of the risk factors for the development of postoperative spinal epidural haematoma. J Bone Joint Surg Br. 2005 Sep;87(9):1248-1252.

2. Cabana F, Pointillart V, Vital J, Sénégas J. Postoperative compressive spinal epidural hematomas. 15 cases and a review of the literature [in French]. Rev Chir Orthop Reparatrice Appar Mot. 2000 Jun;86(4):335-345.

3. Wang $T$, Tian XM, Liu SK, Wang H, Zhang YZ, Ding WY. Prevalence of complications after surgery in treatment for cervical compressive myelopathy: a meta-analysis for last decade. Medicine (Baltimore). 2017 Mar;96(12):e6421. doi: 10.1097/MD.0000000000006421.

4. Amiri AR, Fouyas IP, Cro S, Casey AT. Postoperative spinal epidural hematoma (SEH): incidence, risk factors, onset, and management. Spine J. 2013 Feb;13(2):134-140. doi: 10.1016/j.spinee.2012.10.028.

5. Kaner T, Sasani M, Oktenoglu T, Cirak B, Ozer AF. Postoperative spinal epidural hematoma resulting in cauda equina syndrome: a case report and review of the literature. Cases J. 2009 Jul 16;2:8584. doi: 10.4076/1757-1626-2-8584. 
6. Kao FC, Tsai TT, Chen LH, et al. Symptomatic epidural hematoma after lumbar decompression surgery. Eur Spine J. 2015 Feb;24(2):348-357. doi: 10.1007/s00586-014-3297-8.
7. Ali S, Jabeen S, Pate RJ, et al. Conversion disorder-mind versus body: a review. Innov Clin Neurosci. 2015 May-Jun;12(56):27-33.

This article meets the Accreditation Council for Graduate Medical Education and the American Board of Medical Specialties Maintenance of Certification competencies for Patient Care and Medical Knowledge. 